\title{
Comprehensive literature data-mining analysis reveals a broad genetic network functionally associated with autism spectrum disorder
}

\author{
CHENG XU ${ }^{1 *}$, HONGBAO CAO $^{2,3 *}$, FUQUAN ZHANG $^{4 * *}$ and CHRIS CHEADLE ${ }^{2,5^{* * *}}$ \\ ${ }^{1}$ Department of Magnetic Resonance Imaging, Shanxi Province People's Hospital, Taiyuan, Shanxi 030001, \\ P.R China; ${ }^{2}$ Department of Genomics Research, Elsevier R\&D Solutions, Elsevier Inc., Rockville; \\ ${ }^{3}$ Unit on Statistical Genomics, National Institute of Mental Health/National Institutes of Health, Bethesda, MD 20852, USA; \\ ${ }^{4}$ Wuxi Mental Health Center, Nanjing Medical University, Wuxi, Jiangsu 214151, P.R China; \\ ${ }^{5}$ Department of Rheumatology, Johns Hopkins School of Medicine, Baltimore, MD 21205, USA
}

Received March 28, 2018; Accepted August 1, 2018

DOI: 10.3892/ijmm.2018.3845

\begin{abstract}
Previous studies have indicated that genetic factors are the predominate cause of Autism spectrum disorder (ASD). Nevertheless, to the best of our knowledge, to date no systematic study has summarized these data and provided an objective, complete list of genes with demonstrated associations with ASD. The present study included a literature data mining analysis of $>2,064$ articles including publications from January 2000 to April 2016, which identified 488 ASD target genes. Gene set enrichment analysis (GSEA), sub-network enrichment analysis (SNEA) and network connectivity analysis (NCA) were conducted to assess the functional profile and pathogenic significance of these genes. A total of 2 literature metrics were proposed to prioritize the curated ASD genes with specific significance. This approach resulted in the development of an ASD genetic database. Subsequent analysis indicated that 391 of the 488 genes were enriched in 97 biological pathways $\left(\mathrm{P}<1 \times 10^{-8}\right)$, demonstrating significant functional associations with each other. The majority of these curated ASD genes also serve significant roles in the pathogenesis of other neuropsychiatric disorders. These results suggest that the genetic causes of ASD are within a large network composed of functionally-associated genes. The genetic database, together with the metric scores developed in the present study, provides a basis for future biological/genetic modeling in the field.
\end{abstract}

Correspondence to: Dr Fuquan Zhang, Wuxi Mental Health Center, Nanjing Medical University, 156 Qianrong Road, Wuxi, Jiangsu 214151, P.R China

E-mail: zhangfq@njmu.edu.cn

\section{${ }^{* * * *}$ Contributed equally}

Key words: autism spectrum disorder, literature data mining, gene set enrichment analysis, sub-network enrichment analysis

\section{Introduction}

Autism spectrum disorder (ASD) describes a range of conditions classified as neuronal developmental disorders, including autism, Asperger's syndrome, childhood disintegrative disorder and pervasive developmental disorder. Features of these disorders include social deficits and communication difficulties, stereotyped or repetitive behaviors and interests, sensory issues and in certain cases, cognitive delays (1). While the specific causes of autism spectrum disorders have yet to be identified, it is believed that genetic mutations may be the most significant factor affecting its pathogenesis (2). Other risk factors include prenatal factors, perinatal factors, neuroanatomical abnormalities and certain environmental factors (3-5). While it has been possible to identify general risk factors, it has been more difficult to elucidate specific factors. At present, the prediction of ASD may only be of a global nature in terms of using genetic biomarkers, and therefore requires the use of general markers (6).

There has been an increasing number of articles in the previous decade describing hundreds of genes/proteins associated with ASD, a number of which were suggested as potential disease biomarkers, including interleukin (IL)-6, IL-18, brain-derived neurotrophic factor and RAR related orphan receptor A (7-9). Additionally, several genes [arginine vasopressin (AVP), oxytocin neurophysin 1 prepropeptide (OXT) and insulin-like growth factor 1] have been studied in clinical trials (nos. NCT01093768 and NCT01962870) $(10,11)$. In addition, a number of articles have described genetic changes and alterations in gene expression associated with ASD (12,13). Increased and decreased gene expression levels/activities have been observed (14-16). Multiple genes have also been suggested to affect the pathogenic development of ASD via unknown mechanisms $(17,18)$. A number of studies have suggested a direct functional association between specific gene mutations and ASD. For example, Clipperton-Allen and Page (19) identified that mutations in phosphatase and tensin homolog (PTEN)-encoding negative regulators of the phosphoinositide 3-kinase/protein kinase B/mechanistic target of rapamycin pathway were markedly associated with ASD (19). 
Nevertheless, to the best of our knowledge, no systematic analysis has evaluated the quality and strength of the evidence of these specific genes as a functional network/group underlying the overall biological processes dysregulated in ASD. The present study, instead of focusing on one or two specific genes, aimed to provide a complete description of the genetic map of ASD using a comprehensive literature data-mining review, followed by gene set enrichment analysis (GSEA) and a sub-network enrichment analysis (SNEA) to study the underlying functional profile of the genes identified. We hypothesize that the majority, if not all, of these previously identified genes serve significant roles in the development of ASD, and that the major pathways/gene sets enriched by these genes are those most likely to be functionally associated with the disease.

\section{Materials and methods}

The workflow of the present study was as follows: i) Literature data-mining to discover gene-ASD relations; ii) enrichment analysis on the identified genes to study their pathogenic significance in ASD; iii) network connectivity analysis (NCA) was used to test for direct functional association between the top identified genes. A genetic database (ASD_GD) has been developed and deposited into an open source 'Bioinformatics Database' (http://database. gousinfo.com). The publicly downloadable version of the database is available at http://gousinfo.com/database/Data Genetic/ASD_GD.xlsx. The database includes 488 genes with metric scores, 97 pathways and 93 diseases that were associated with ASD. The 97 pathways were identified through a GSEA approach using the ASD target genes as input against the Gene Ontology (GO) and Pathway Studio Ontology. The 93 diseases were identified using a SNEA process. Both the GSEA and SNEA were conducted using Pathway Studio, where the enrichment P-values and Jaccard similarity $\left(J_{s}\right)$ were provided in the analysis results. For each ASD-gene association, there was information regarding the supporting references, including titles and specific sentences where the association had been identified. For more information regarding the database, please refer to 'ASD_GD $\rightarrow$ Database Note' (http://gousinfo.com/database/Data_Genetic/ASD_GD.xlsx), where the subsequent update information is also included.

Literature data mining and article selection criteria. In the present study, a literature data-mining analysis was performed for all articles describing gene-ASD associations available in the Pathway Studio database (www.pathwaystudio.com) updated until April 2016. The Pathway Studio database contains over $6.1 \mathrm{M}$ unique associations supported by $\geq 35$ million references. The literature data-mining analysis was conducted using Elsevier's proprietary MedScan natural language processing (NLP) system of the Pathway Studio software as described previously (20), in which essential facts are extracted according to predefined fact types in the form of information triplets (subject-verb-object). Domain ontologies are developed in order to identify types, properties and inter-associations of relevant entities in the biomedical literature (21). The data extracted included information on proteins/genes, small molecules, cell types and diseases represented as entities, and their interactions were defined by association types including binding, regulation and expression. The result was a database of genomic and proteomic information, with a specific focus on how the proteins, cell processes and small molecules interact, modify and regulate each other in pathways and networks. Search results were obtained from the Pathway Studio database (www. Pathwaystudio.com), including a full list of gene names, the types of associations and the bibliographic information of the supporting articles; for example, the titles and specific sentences where the associations had been identified, allowing for the calculation of the article metric scores described subsequently.

Literature metrics analysis. For literature metrics analysis, three 'weights' for each article and two scores for each gene-disease association were proposed.

Definition of three weights for an article. i) The quality weight $(\mathrm{QW})$. The QW of an article was defined as Eq. 1.

$$
\mathrm{QW}=\mathrm{e}^{-\beta \times \text { [publication age (PubAge)-1] }}(\text { Eq. 1); }
$$

where PubAge $=$ current date - publication date +1 years; $\beta=0.23$, such that $\mathrm{QW}=1$ for literature published in a current year and $\mathrm{QW}=0.1$ if it was published 10 years previously.

ii) The Citation Weight $(\mathrm{CW})$. The $\mathrm{CW}$ of a reference was defined as Eq. 2.

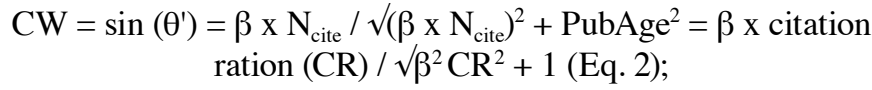

where the $C R=$ number of citations/PubAge. $\beta$ was set to control the initial values of $C W . \beta=1 / \sqrt{ } 3$, so that $C W=0.5$ when $\mathrm{CR}=1\left(\mathrm{~N}_{\text {cite }}=\right.$ PubAge $)$.

iii) The Novelty Weight (NW). The NW of a reference was defined as Eq. 3, where;

$\mathrm{NW}=0$ or 1 (0: PubAge $>$ AgeNovel; 1 : otherwise) $($ Eq. 3$)$

The Novelty Age threshold was specified by AgeNovel in Eq. 3. This represented the maximum age for all references in order for that association to be considered novel. In this study, we set AgeNovel=1 to surface only these identified in the current year as novel markers.

Quality Score (QScore) for a gene-disease association. The QScore for a literature-based gene-disease association was defined as Eq. 4, where:

$$
\mathrm{QScore}=\operatorname{sum}\left(\mathrm{CW}_{\mathrm{i}}+\mathrm{QW}_{\mathrm{i}}\right) / 2, \mathrm{i}=1,2, \ldots, \mathrm{n}(\mathrm{Eq} .4)
$$

where $\mathrm{CW}_{\mathrm{i}}$ and $\mathrm{QW}_{\mathrm{i}}$ are the weights for the reference $\mathrm{i}(\mathrm{i}=1,2$, etc.), and $n$ represents the total number of references supporting an association.

The Novelty Score (NScore) was defined as Eq. 5, where:

$$
\begin{gathered}
\text { NScore }=\left[\operatorname{sum}\left(\mathrm{CW}_{\mathrm{i}}+\mathrm{NW}_{\mathrm{i}}\right)\right]^{*}\left[\mathrm{NW}_{1} * \mathrm{NW}_{2} * \ldots * \mathrm{NW}_{\mathrm{n}}\right], \\
\mathrm{i}=1,2, \ldots, \mathrm{n}(\text { Eq. 5) }
\end{gathered}
$$


Table I. Top 14 genes selected by QScore.

\begin{tabular}{|c|c|c|c|c|c|c|c|}
\hline Gene symbol & QScore & NScore & Citation number & RefNum & Age, years & EScore & Number of pathways \\
\hline SHANK3 & 80.0 & 0 & 5,397 & 139 & 10 & 320.5 & 18 \\
\hline MECP2 & 72.6 & 0 & 5,320 & 108 & 15 & 325.9 & 22 \\
\hline PTEN & 64.9 & 0 & 4,268 & 100 & 12 & 432.4 & 28 \\
\hline NLGN3 & 52.4 & 0 & 3,200 & 74 & 14 & 249.6 & 18 \\
\hline NLGN4X & 45.6 & 0 & 2,655 & 61 & 14 & 255.2 & 14 \\
\hline OXT & 44.4 & 0 & 3,920 & 88 & 14 & 161.7 & 10 \\
\hline OXTR & 42.1 & 0 & 3,631 & 64 & 9 & 194.8 & 11 \\
\hline NRXN1 & 39.3 & 0 & 2,624 & 63 & 9 & 312.8 & 20 \\
\hline SHANK2 & 34.4 & 0 & 1,784 & 61 & 8 & 303 & 15 \\
\hline CNTNAP2 & 33.5 & 0 & 2,460 & 53 & 9 & 220.3 & 12 \\
\hline SLC6A4 & 17.9 & 0 & 742 & 30 & 13 & 204.9 & 11 \\
\hline TSC2 & 17.9 & 0 & 1,723 & 27 & 13 & 183.7 & 13 \\
\hline TSC1 & 16.4 & 0 & 1,715 & 20 & 11 & 47.1 & 4 \\
\hline CD38 & 14.7 & 0 & 713 & 22 & 7 & 82 & 5 \\
\hline
\end{tabular}

QScore, quality score; NScore, novelty score; RefNum, number of reference; EScore, enrichment score.

where $\mathrm{n}$ represents the total number of references supporting a gene-disease association, and $\mathrm{CW}_{\mathrm{i}}$ and $\mathrm{QW}_{\mathrm{i}}$ are the weights for the ${ }^{\mathrm{i}} \mathrm{i}$ ' reference.

GSEA. To fully elucidate the underlying functional profile and the pathogenic significance of the identified genes, GSEA and SNEA were performed as described previously (22) on the entire gene list ( $n=488$ genes).

Pathway enrichment analysis. When considering a disease associated with a number of genetic pathways, the gene-wise Enrichment Score (EScore) for the gene $\mathrm{k}(\mathrm{k}=1,2,3$, etc.) within a gene set was defined as Eq. 6 where;

$$
\begin{gathered}
\text { EScore }=\operatorname{sum}\left[-\log _{10}\left(\text { pValue }_{\mathrm{i}}\right)\right] / \max \left[-\log _{10}\left(\text { pValue }_{\mathrm{i}}\right)\right], \\
\mathrm{i}=1,2, \ldots, \mathrm{n}(\text { Eq. 6) }
\end{gathered}
$$

where 'P-value ${ }_{i}$ ' is the enrichment score of the ' $\mathrm{i}^{\text {th }}$, pathway within the gene set, ' $n$ ' represents the total number of pathways and ' $\mathrm{m}$ ' represents the number of pathways including the ' $\mathrm{k}$ th' gene.

\section{Results}

Summary of literature data mining analysis. In the present study, a literature data-mining analysis of 2,064 articles was performed and identified a total of 488 genes directly associated with ASD in the scientific literature. According to the categories of gene-ASD associations, the 2,064 articles were identified in 6 different classes: i) Biomarker (0.3\%); ii) Clinical Trial (0.5\%); iii) Genetic Change (78\%); iv) Quantitative Change (6.6\%); v) Regulation (14.2\%); and, vi) State Change $(0.4 \%)$. Overall, for the 488 genes, $0.8 \%$ were demonstrated to have a 'Biomarker' association with the disease, $0.5 \%$ genes with 'Clinical Trial', 59.2\% with 'Genetic Change', 15.9\% with 'Quantitative Change', 22\% with 'Regulation' and $1.7 \%$ with 'State Change'. One gene may present multiple associations with ASD. An example of this was identified for the AVP gene, which was identified in four separate association types: 'Genetic Change'; 'Quantitative Change'; 'Clinical Trial'; and 'Regulation'. A full list of genes and supported associations may be located in the 'ASD_GD-Ref for Related Genes' database.

Altogether, $23.7 \%$ genes were demonstrated to have multiple associations with ALS. Specifically, 76.3\% genes presented 1 type of association, $17.4 \%$ with $2,4.9 \%$ with 3 and $1.4 \%$ with 4 , as indicated in Figs. 1 and $2 \mathrm{~A}$ demonstrates the publication date distribution of the 2,064 articles, including literature data from the previous 16 years (2000-2016), with the number of novel genes identified each year presented in Fig. 2B. Notably, the ASD-associated articles had an average PubAge of only 3.6 years. In the present study, the PubAge was defined as: Current year-publication date +1 year. In addition, a significant increase in the total number of publications was observed in the past 10 years, particularly in the period subsequent to 2012, with the highest number of novel genes identified during this time period (Fig. 2B).

Literature metric scores and rankings. Table I summarizes the top 14 genes of the entire set of gene-disease associations for ASD by QScore. The NScore for these genes was 0 as, in every case, the age of their oldest publication exceeded the calculated threshold for novelty (set at 1 year). As expected, the QScore for these genes was similar to their number of associated references, indicating that the relations supported by a large number of references were less likely to be affected overall by variations in aggregate PubAge or by the cumulative citation numbers of the underlying articles. In other words, a high reference number was the primary driver for higher QScores, suggesting that QScores may be the most useful 


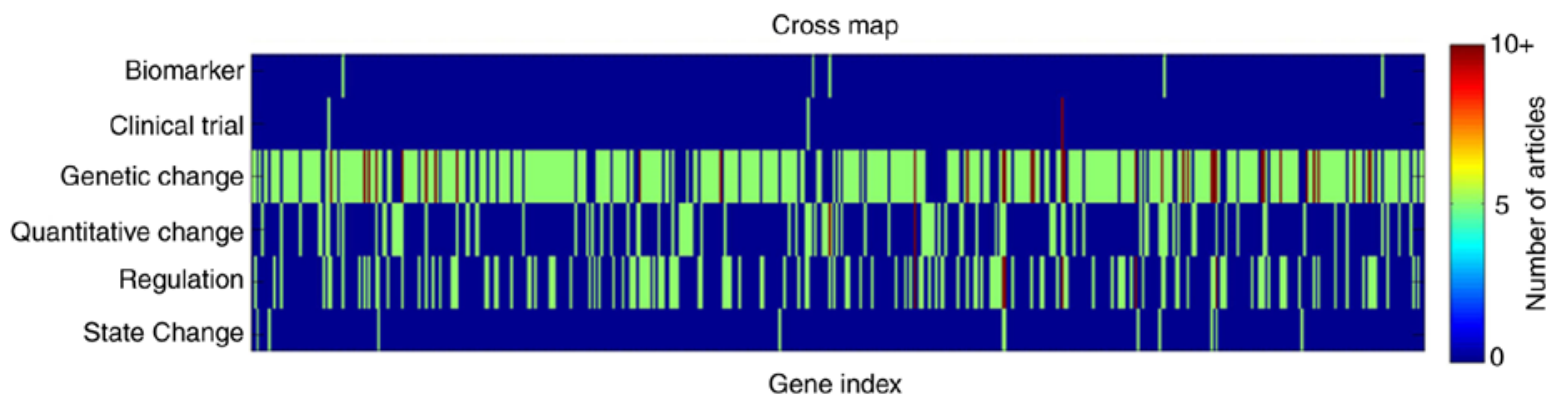

Figure 1. Distribution of 488 genes among different association types. The X-axis represents the gene index (gene 1-488). The Y-axis represents the types of gene-ASD associations. The color at a coordinate $(x, y)$ represents the number of articles reporting a ' $y$ ' association type for the gene ' $x$ '. The legend on the right denotes the number-color correspondence; the continuous change of color from blue to red represents a change of number from 0 to 10 , where 0 signifies that there are no articles supporting the association. ASD, autism spectrum disorder.
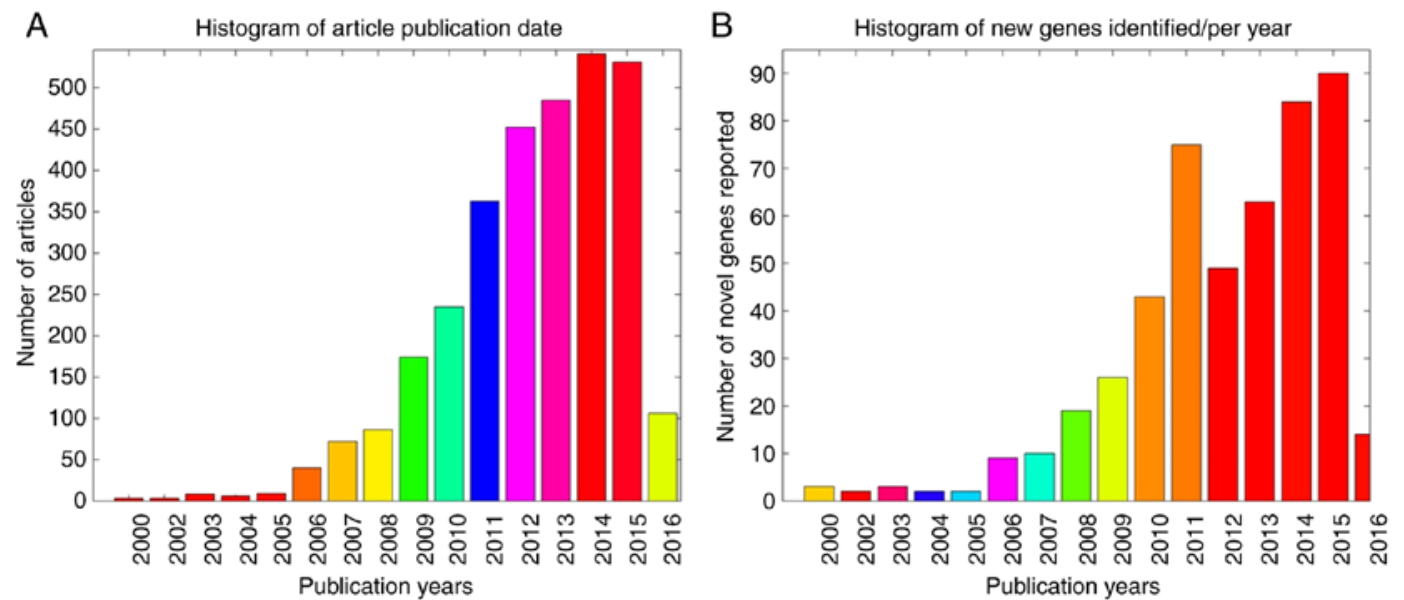

Figure 2. Histogram of the publications describing gene-disease associations between 488 unique genes and autism spectrum disorder. (A) Histogram of article publication date. (B) Histogram of the number of novel genes identified in each year.

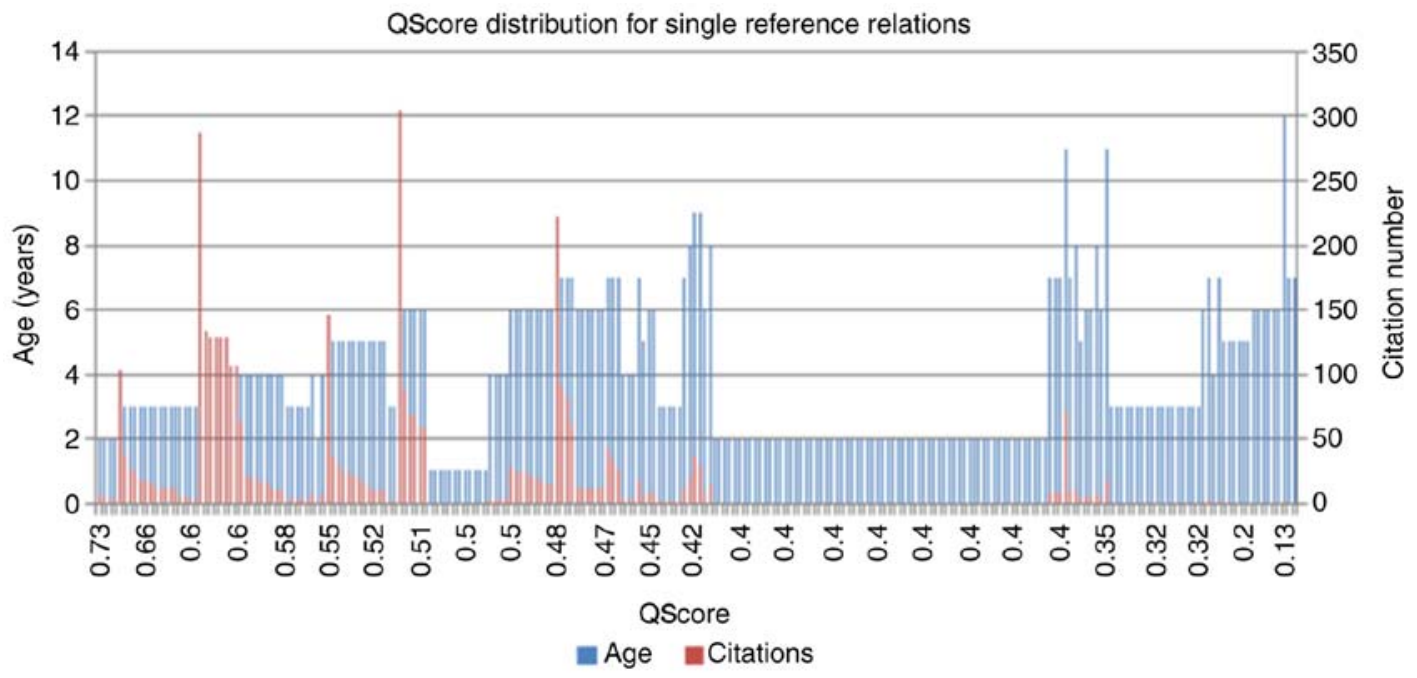

Figure 3. All autism spectrum disorder-gene single reference associations rank-ordered by QScore. A higher QScore for a given association was derived from a combination of a recent publication date (blue bar) and high citation rate (red bar). QScore, quality score.

indicator for distinguishing associations at the low end of the reference distribution, where the factors of age and $\mathrm{CW}$ will be able to provide additional discriminatory power. An example of this is provided in Fig. 3, which indicates the distribution of the PubAge and the citation number compared with the calcu- lated QScores for all single reference associations among the ASD literature-associated genes. The single reference genes comprise almost one-half of all the ASD-associated genes $(236 / 488 \times 100=48 \%)$, and therefore it is important and useful to provide a method for identifying the strongest candidate 
Table II. Top 14 genes selected by NScore.

\begin{tabular}{|c|c|c|c|c|c|c|c|}
\hline Gene symbol & QScore & NScore & Citation number & RefNum & Marker age & EScore & Number of pathways \\
\hline CYP2D6 & 2 & 4 & 0 & 4 & 1 & 9.1 & 1 \\
\hline EP300 & 1.5 & 3 & 0 & 3 & 1 & 189.3 & 13 \\
\hline UNC80 & 0.5 & 1 & 0 & 1 & 1 & 5.6 & 1 \\
\hline TP53 & 0.5 & 1 & 0 & 1 & 1 & 126 & 12 \\
\hline SLC9A6 & 0.5 & 1 & 0 & 1 & 1 & 69 & 3 \\
\hline SETD1A & 0.5 & 1 & 0 & 1 & 1 & 0 & 0 \\
\hline PIK3CD & 0.5 & 1 & 0 & 1 & 1 & 0 & 0 \\
\hline NFE2L2 & 0.5 & 1 & 0 & 1 & 1 & 15.9 & 2 \\
\hline IL18 & 0.5 & 1 & 0 & 1 & 1 & 52.1 & 6 \\
\hline HTR3A & 0.5 & 1 & 0 & 1 & 1 & 182.5 & 9 \\
\hline CX3CR1 & 0.5 & 1 & 0 & 1 & 1 & 64.5 & 4 \\
\hline CUX1 & 0.5 & 1 & 0 & 1 & 1 & 0 & 0 \\
\hline CASP1 & 0.5 & 1 & 0 & 1 & 1 & 148.1 & 9 \\
\hline CAPRIN1 & 0.5 & 1 & 0 & 1 & 1 & 38.1 & 1 \\
\hline
\end{tabular}

QScore, quality score; NScore, novelty score; RefNum, number of reference; EScore, enrichment score. NScore here is based on a Novelty Age threshold=1; any genes that were identified prior to 2016 will have an NScore of 0 . In this study there were 14 genes identified in 2016.

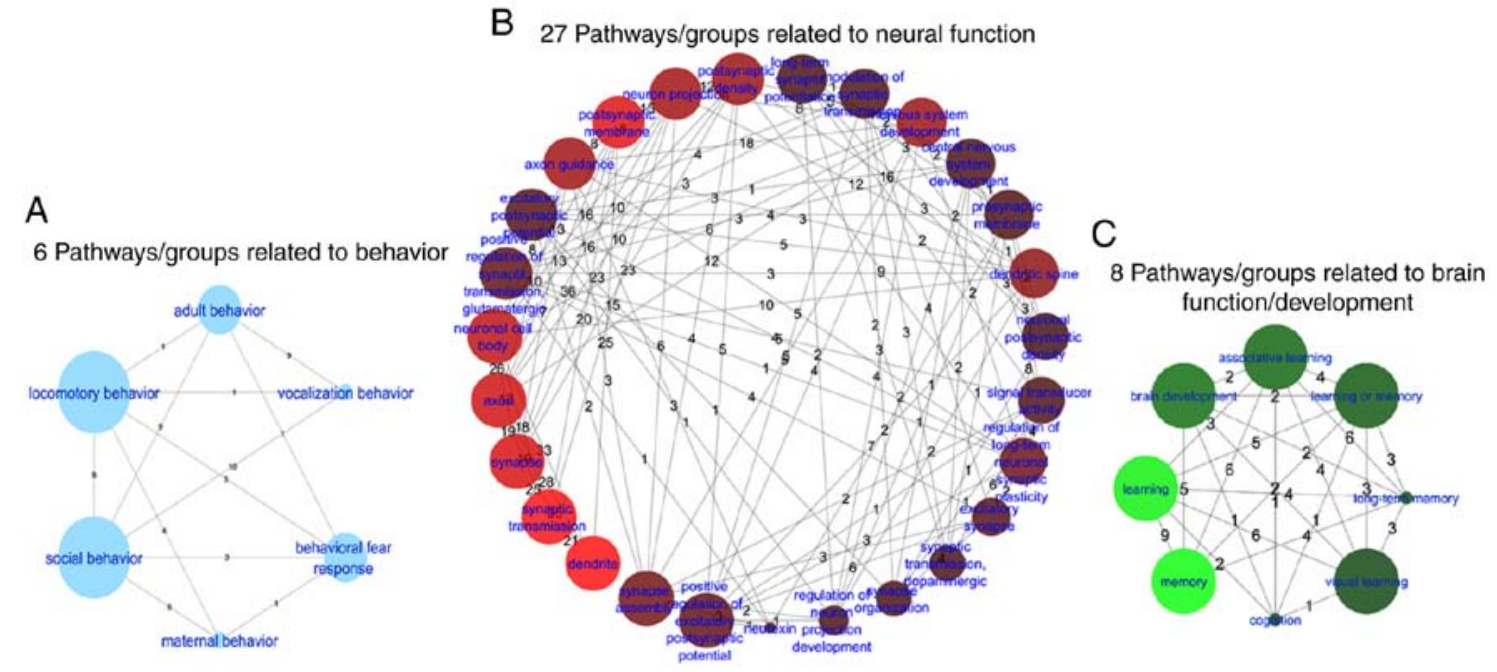

Figure 4. Pathways for (A) behavior, (B) neural function and (C) brain function/development. In all networks, the weight of the line (number attached to the line) between two nodes is the number of genes shared by those two pathways. The size of the nodes represents the P-value (- $\log _{10}$ transformed) of the enriched pathway. The brighter the color of a node, the higher the number of the pathways connected to the node.

genes in this group. As observed in Fig. 3, the association presenting the highest QScores were these supported by a high-number of references with a strong number of citation and recent publication dates. Older publications with fewer citations were correspondingly penalized by this method and produced lower relative QScores. The apparent absence of demonstrated reproducibility for the associations within this group, for example, they remained single reference associations over a longer period of time, suggests that these associations should be analyzed with caution, prior to their acceptance as fully reliable.

On the other hand, NScores provided a useful method for evaluating the most recently observed gene-disease associations. While in practice, the user is able to set the Novelty Age threshold, which represented the maximum age for all references in order for that association to be considered novel, to any number of years desired. In the present study, the most stringent threshold of 1 year was used; therefore, only associations with publications in 2016 were selected and this resulted in the identification of a total of 14 novel genes (Table II). Table II also presents the QScore for each of these 14 genes The genes for cytochrome P450 family 2 subfamily D member 6 (CYP2D6) and E1A binding protein P300 (EP300) exhibited the two highest NScores, as a result of the number of references supporting the relation between these two genes and ASD (4 and 3 references for CYP2D6 and EP300, respectively), indicating that they should be prioritized among the novel candidate genes identified for future study. Notably, EP300 
Table III. Top 10 pathways/groups enriched for all autism spectrum disorder-associated genes.

\begin{tabular}{lcccccc}
\hline Name & $\begin{array}{c}\text { Gene } \\
\text { ontology ID }\end{array}$ & $\begin{array}{c}\text { Number of } \\
\text { entities }\end{array}$ & $\begin{array}{c}\text { Number of } \\
\text { overlapping genes }\end{array}$ & $\begin{array}{c}\text { P-value } \\
\text { Qimilarity }\end{array}$ & $\begin{array}{c}\text { Q-value } \\
\text { simeard }\end{array}$ \\
\hline Dendrite & 0030425 & 396 & 68 & $5.72 \times 10^{-40}$ & $1.91 \times 10^{-37}$ & 0.081 \\
Synaptic transmission & 0007268 & 472 & 72 & $1.18 \times 10^{-37}$ & $2.95 \times 10^{-35}$ & 0.079 \\
Response to drug & 0017035 & 509 & 74 & $3.02 \times 10^{-37}$ & $6.04 \times 10^{-35}$ & 0.078 \\
Postsynaptic membrane & 0045211 & 227 & 49 & $8.27 \times 10^{-34}$ & $1.38 \times 10^{-31}$ & 0.071 \\
Synapse & 0045202 & 466 & 65 & $1.36 \times 10^{-32}$ & $1.95 \times 10^{-30}$ & 0.072 \\
Axon & 0030424 & 318 & 54 & $1.5 \times 10^{-31}$ & $1.87 \times 10^{-29}$ & 0.07 \\
Social behavior & 0035176 & 52 & 27 & $1.34 \times 10^{-30}$ & $1.49 \times 10^{-28}$ & 0.051 \\
Neuronal cell body & 0043025 & 466 & 61 & $4.77 \times 10^{-29}$ & $4.34 \times 10^{-27}$ & 0.067 \\
Memory & 0007613 & 76 & 29 & $7.07 \times 10^{-28}$ & $5.89 \times 10^{-26}$ & 0.052 \\
Response to organic cyclic compound & 0014070 & 253 & 46 & $1.28 \times 10^{-27}$ & $9.86 \times 10^{-26}$ & 0.064 \\
\hline
\end{tabular}

For each gene set, the P-value was calculated using Fisher's exact test against the hypothesis that a randomly selected gene group of same size (488) may generate a same or higher overlap with the corresponding gene set $(\mathrm{Q}=0.001$ for false discovery rate correction). The Jaccard similarity $\left(J_{s}\right)$ is a statistic used for comparing the similarity and diversity of sample sets, which is defined by $J_{s}(A, B)=\frac{A \cap B}{A \cup B}$, where $A$ and $B$ are two sample sets.

Table IV. Top 10 disease sub-networks enriched for all autism spectrum disorder-associated genes.

\begin{tabular}{lcccc}
\hline Gene set seed & Total number of neighbors & Overlap & P-value & Jaccard similarity \\
\hline Schizophrenia & 1,230 & 234 & $1.6 \times 10^{-176}$ & 0.16 \\
Epilepsy & 756 & 164 & $1.1 \times 10^{-127}$ & 0.15 \\
Intellectual disability & 821 & 166 & $4.9 \times 10^{-124}$ & 0.15 \\
Seizures & 832 & 164 & $1.7 \times 10^{-120}$ & 0.14 \\
Cognitive impairment & 409 & 126 & $3.6 \times 10^{-117}$ & 0.17 \\
Bipolar disorder & 490 & 124 & $2.2 \times 10^{-103}$ & 0.15 \\
Depressive disorder, major & 439 & 115 & $2.1 \times 10^{-97}$ & 0.14 \\
Alzheimer disease & 1,312 & 169 & $3.6 \times 10^{-93}$ & 0.1 \\
Cognition disorders & 322 & 95 & $2.8 \times 10^{-85}$ & 0.13 \\
Anxiety & 379 & 99 & $3.5 \times 10^{-83}$ & 0.13
\end{tabular}

exhibited a considerably higher EScore and participated in a greater number of ASD-associated pathways compared with CYP2D6, indicating a greater concordance, at least at the level of pathway involvement, with previously studied ASD genes and proteins. This may also indicate that CYP2D6 is representative of a novel mechanism not previously studied in association with ASD. Other high Escore candidates among the novel genes included tumor protein 53 (TP53), 5-hydroxytriptamine receptor $3 \mathrm{~A}$ (HTR3A) and caspase (CASP1).

Enrichment analysis. The full list of 97 pathways/gene sets enriched with a minimum $\mathrm{P}<1 \mathrm{E}-8$ (391 unique genes) is available in the 'ASD_GD-Related Pathways' database, where 58 pathways/gene sets are demonstrated to be enriched with $\mathrm{P}<1 \times 10^{-10}$ (364 unique genes), and 16 were enriched with $\mathrm{P}<1 \times 10^{-20}$ (272 unique genes).

Among the 97 pathways/gene sets enriched, 27 pathways/gene sets that were directly associated with neural function (260 unique genes), 8 with brain function/development (103 unique genes) and 6 with behavior (112 unique genes) were determined (Fig. 4). In addition, a single gene ontology (GO) term associated with aging (30 unique genes; $\mathrm{Q}<7$.$) was identified. The top 10$ pathways/groups enriched by all genes with $\mathrm{P}<1.0 \times 10^{-25}$ (221 unique genes) are summarized in Table III. The Jaccard similarity $\left(J_{s}\right)$ statistic is an alternative method for comparing the similarity and diversity of sample sets.

In addition to GSEA, a SNEA was performed. SNEA, an extension of GSEA, constructs gene sets using the global network of protein-disease regulation as represented in the Pathway Studio database (23). The proteins linked to the diseases identified by GAEA presented significant overlap with the 488 ASD target genes. In the present study, enrichments were calculated using diseases as the gene set seeds for this specific group of genes/proteins, for the purpose of identifying patterns of pathogenic significance to other disorders 


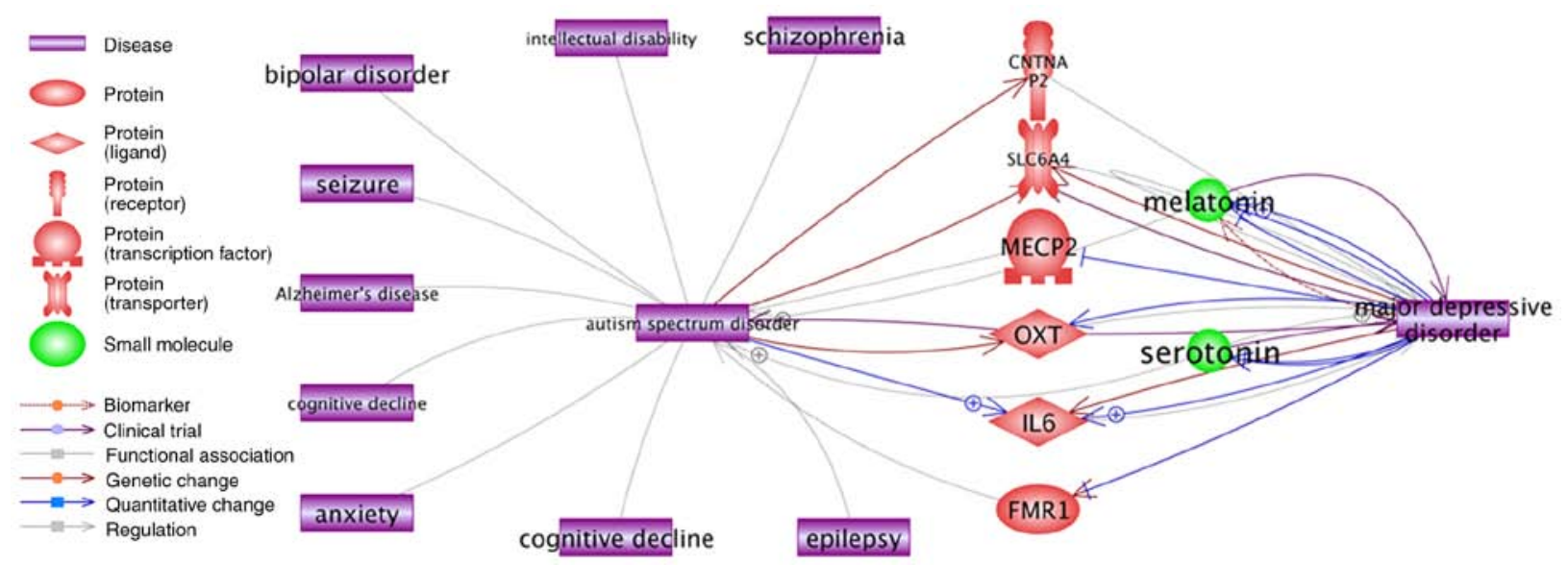

Figure 5. Disease-disease interaction network between autism spectrum disorder and the top 10 mental disorders from the sub-network enrichment analysis. CNTNAP2, contactin associated protein like 2; SLC6A4, solute carrier family 6 member 4; MECP2, methyl CpG-binding protein 2; OXT, oxytocin neurophysin 1 prepropeptide; IL6, interleukin 6; FMR1, fragile X mental retardation 1.
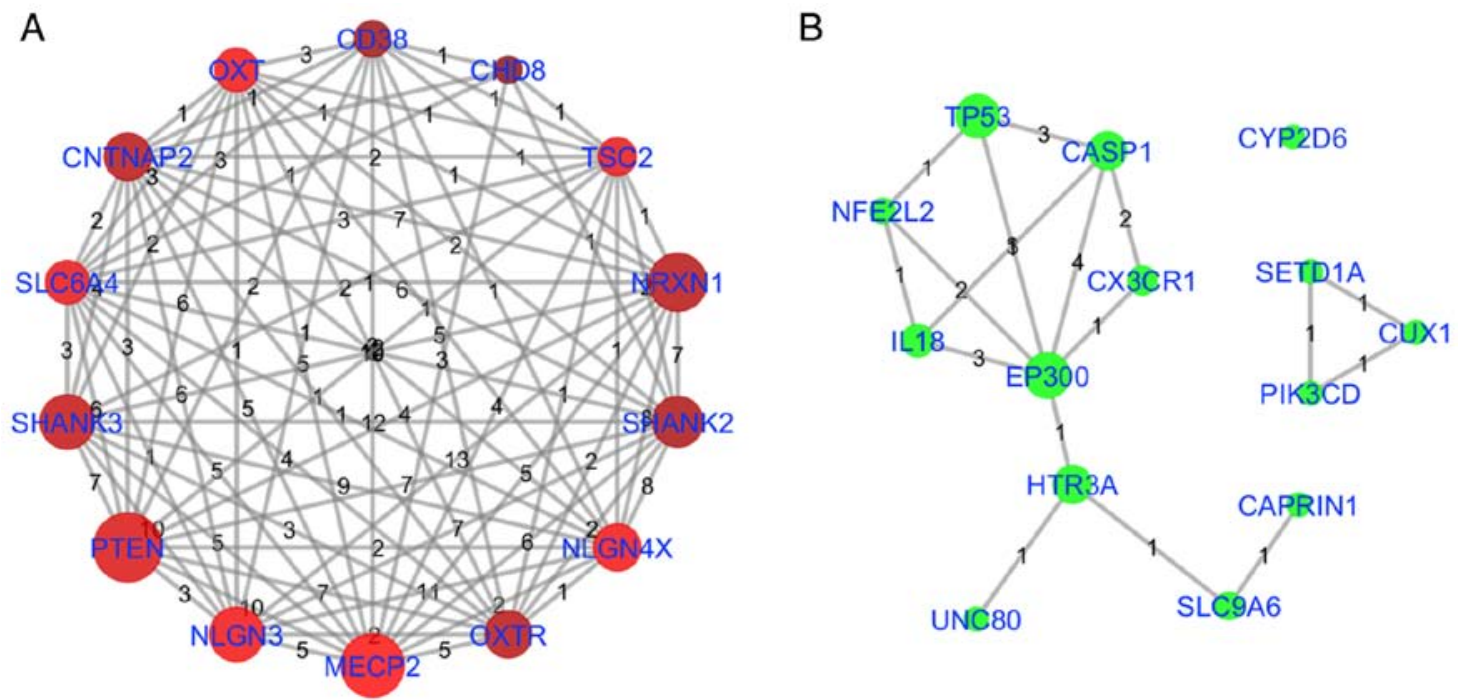

Figure 6. Gene network of top 14 genes by NScore and QScore. (A) QScore group; the brighter the color (red), the higher the QScore. (B) NScore group; the brighter the color (green), the higher the NScore. The size of each node represents the number of pathways (from the pathway enrichment analysis) a gene was identified in. The weight of the line between any two nodes is the number of pathways shared by those two genes. NScore, novelty score; QScore, quality score. Due to high number of edges, the weights on the edge may not differentiable. Please refer to ASD_GD $\rightarrow$ Related Genes, where the related pathways of each gene have been provided.

potentially associated with ASD. The complete list of results is provided in the 'ASD_GD-Related Diseases' database. Table IV presents the top 10 disease-associated sub-networks enriched with a minimum $\mathrm{P}<1 \times 10^{-80}$.

From Table IV, it was observed that a number of the established ASD-associated genes were also identified as highly significant (as a subgroup) in other mental health-associated diseases, with a large percentage of overlap (Jaccard similarity $\geq 0.1$ ). A direct functional association for 9 of the 10 enriched disease types was also identified in the Pathway Studio literature database between these mental disorders and ASD (Fig. 5). Notably, although there was no direct association between ASD and Major depressive disorder, indirect associations were readily demonstrated through certain high QScore ASD genes including methyl CpG-binding protein 2 (MECP2) (QScore=71.4), OXT (QScore=42.1), and contactin associated protein-like 2 (QScore=35.3).
Prioritizing new literature information using NScores. One of the challenges in evaluating previous gene-disease associations in the literature is the ability to rapidly sort through information to identify the most notable, reliable and informative additions to previously studied and accepted observations. As previously stated, a high QScore ranking may provide a rapid and considered sense of the most reliable data, primarily in terms of multiple reference support, but also secondarily by the aggregate PubAge and CW.

Conversely, observations that are supported by a limited number of references (almost one-half of the ASD-associated genes in the present study were supported by a single reference) were ranked conveniently by PubAge and CW (Eq. 5). Unique cases arise when the association is so novel, as demonstrated by the genes/proteins in Table II, that citation data is not necessarily available, leaving only the criterion of the number of references to evaluate the relevance of a gene/protein-centered 
disease association. This functions to highlight associations that already have multiple source articles, as previously identified for EP300 and CYP2D6, but does not address the majority of novel gene associations supported by only a single reference. A way to evaluate these novel associations, in the absence of other literature metric data, may be to map them to the pathways domain already defined by the hundreds of higher QScore genes/proteins. This information was generated using all the genes/proteins demonstrating associations with ASD, as summarized in Table III and Fig. 4, and as illustrated in Fig. 6. Fig. 6A describes the NCA for the top 14 highest QScore genes, indicating significant mutual pathway participation. Fig. 6B indicates the NCA for the top 14 NScore genes using the same pathways. As highlighted previously, EP300 was identified as significant in terms of pathway participation (included in 13 out of 97 pathways) and NScore (NScore=3). TP53 and CASP1 also demonstrated marked pathway involvement and NScore connectivity. HTR3A indicated marked pathway involvement and limited NScore connectivity. However, HTR3A was associated with the novel AD genes Unc-80 homolog, NALCN channel complex subunit, which encodes a leak channel that serves an important role in the establishment and maintenance of resting membrane potentials in neurons,mutations of which are associated with intellectual disability, and SLC9A6, a sodium-hydrogen exchanger involved with synapse activity, mutations of which are associated with mental retardation, and therefore may be of signifciance in future studies. Conversely, CYP2D6 exhibited a high NScore and weak associations with pathways previously studied in association with autism, and may have been identifed using this analysis primarily as a result of the effects of CYP2D6 gene polymorphisms on the clearance rates of the drug risperidone used in the treatment of children with ASD (24).

\section{Discussion}

In the present study, a literature data-mining analysis was performed on 2,064 articles dating from January 2000 to April 2016, and identified 488 unique genes/proteins associated with ASD. Based on the approach, an ASD_GD database was developed. Within the ASD_GD database, the complete gene list together with the literature references and enrichment metrics scores are provided. Results from GSEA and SNEA provide evidence of a functional association between the majority of these genes at the pathway level and may assist in identifying which genes/proteins may serve important roles in the pathogenesis of ASD.

As an automated literature data mining approach, the Pathway Studio NLP semantic analysis technique is highly effective and efficient in dealing with large amounts of data in literature data-mining analyses (25). In the ASD_GD-Ref for Related Genes database, the detailed information of all the 2,064 articles studied, including the sentences from which a specific association was extracted, is included. The results of the present study are intended to provide an overall view of the current state of genetic studies in ASD, mitigate occasional errors and provide a basis for additional biological/genetic studies.

From ASD_GD to Related Genes the number of references for a given association can be observed to be closely correlated with its QScore suggesting that the QScore may be the most useful indicator in analyzing associations with limited numbers of references, on the basis of CW and PubAge. Similarly, CR is also closely correlated with the number of overall references for a relation $\left(\mathrm{R}^{2}=0.94\right.$ across all 488 ASD-associated genes/proteins). As expected, PubAge is also loosely correlated with reference number $\left(A S D \_G D \rightarrow\right.$ Publication Age; included in the downloadable version of the datatbase) but with certain notable exceptions; for example, the autism-associated chromatin modifier chromodomain helicase DNA binding protein 8 (CHD8) gene (13) (QScore=20.8). The first publication associating CDH8 with autism is only 5 years old (26), but has already been highly cited (1,974 citations) and an ASD-like behavioral phenotype, including increased anxiety, repetitive behavior and altered social behavior, was demonstrated in mice heterozygous for CHD8 mutations (27).

The present study also observed that the majority of genes identified by the literature data-mining analysis were included in pathways previously implicated with ASD. Specifically, $391 / 488$ total genes/proteins were included in at least one of the top 97 enriched pathways, and 221/488 genes/proteins in the top 10 pathways. A number of these 97 pathways are potentially functionally associated with ASD, including 21 neural system, 7 brain function, 3 behavior, and one aging pathway. We hypothesize that the majority of these genes identified in the literature, in particular the ones that were identified from significantly enriched pathways, are functionally associated with ASD. Although there may be false positives included within individual studies, it is less likely that as a group, these genes have been identified as being disrupted in a disease or disorder by chance.

The analysis in the present study did not specifically focus on single genes; instead, it was noted that the 488 unique genes/proteins identified were not equal in terms of publication frequency,citation rate of the underlying articles $(\mathrm{CW})$ or by their novelty (NScore). Using the proposed literature quality metrics scores including QScore, the genes were ranked according to several different criteria and significance measures, and the top 14 genes were selected for subsequent analysis. For example, SH3 and multiple ankyrin repeat domains 3, MECP2, PTEN, neuroligin 3 and neuroligin 4, X-linked are the top 5 genes that were most often replicated in previous studies (with highest QScores), suggesting that their associations with ASD are well-studied. These 5 particular genes/proteins were most enriched for the GO term (GO:0035176) for 'Social Behavior' [false discovery rate (FDR) $Q=0.0015]$ (26), additionally supporting the association of these high QScore genes/proteins with the clinical presentation of social-behavioral characteristics believed to comprise the broad autism phenotype. Notably, 25/48 genes/proteins of this particular GO term category were also identified among the 488 unique ASD-associated genes/proteins $\left(\mathrm{P}=5.2 \times 10^{-29}\right)$. Alternatively, the top 2 genes identified by NScore, namely CYP2D6 and EP300, were previously analyzed multiple times in 2016 . These data suggest that these genes are likely to possess significant biological relevance to the disease.

Additionally, it was noted that for the top 97 pathways enriched with 443/488 unique gene/proteins, certain genes were identified in multiple significantly enriched pathways, thereby generating high pathway enrichment EScores. The 
top 5 genes by EScore included glutamate ionotropic receptor NMDA type (GRIN) subunit 1 (42/97 pathways), GRIN subunit 2A (38/97), dopamine receptor (DR) D1 (36/97), GRIN subunit 2B (GRIN2B; 38/97) and DRD2 (31/97), which were revealed to serve multiple roles within different genetic pathways associated with ASD, suggesting their biological significance in the disease. Notably, this group of dopamine and glutamate ionotropic receptors was most markedly associated, by GO term enrichment analysis, with the response to amphetamine (FDR Q=0.008) (28) and its effects on visual learning and cognition. This group of genes, with the exception of GRIN2B (QScore=13.6), exhibited low QScores $(<2)$ indicating that they have not yet been well studied within the context of autism and perhaps, given the data from the present study, may warrant additional analyses. The combination of NScore and EScore has been suggested to be effective in identifying highly relevant genes/proteins that may otherwise have been overlooked either due to low publication frequency and/or old publication date. The exploration of the predictive ability of EScore, in particular, for identifying novel genes/proteins prior to publication is important, and is currently being investigated by our study group.

In addition to GSEA, a SNEA was also performed, which was implemented in Pathway Studio using master casual networks generated from $>6.5$ million associations derived from $>4$ million full-text articles and 25 million PubMed abstracts. The ability of the Pathway Studio automated NLP technology to quickly update the terminologies and linguistics rules used by the NLP systems ensures that novel terms may be captured rapidly following their entrance into regular use in the literature, and updates of literature results occur on a weekly basis. This extensive database of interaction data provides high levels of confidence when interpreting experimentally-derived genetic data against the background of previously published results. The SNEA results of the present study demonstrated that the majority of the 488 unique genes/proteins (>90\%) were also identified to have regulatory roles in other disparate mental health disorders, including schizophrenia, epilepsy and cognitive impairment, alongside marked associations with ASD (29-31). A total of 9 out of 10 of the most significantly enriched ASD gene/protein associated diseases were also demonstrated to be directly functionally associated with ASD.

Results from the literature data-mining analysis of the present study demonstrate that the majority of the 488 unique gene/proteins identified by this method exhibited multiple types of associations with ASD. The literature and enrichment metrics employed identified key genes with specific significance to ASD. In addition, NCA and GSEA results suggested that these genes/proteins serve significant roles within a network of associated genes contributing to the pathogenesis of ASD. Results from the sub-network analysis indicate that these same genes have significant cross-over effects with other mental disorders.

Notably, the data analysis approach proposed in the present study may be applied to updated ASD literature data. Consequently, an update of the ASD_GD database has been generated following the same procedure with updated ASD literature data (up to June 2018). By comparing the two databases (e.g., the current ASD_GD and the one updated in June
2018), it has been demonstrated that the approach outlined in the present study is applicable to other literature data mining techniques within disease-gene association studies.

In conclusion; ASD is a complex disease whose genetic causes are associated with a network composed of a large group of genes. Literature data-mining analysis together with GSEA, SNEA and NCA methods may serve as an effective approach in identifying these potential target genes. Comparative analysis using a similar approach with multiple types of mental disorders may be useful for identifying not only the most relevant and significant disease-specific gene targets but may also serve as a reliable method for identifying significant areas of homology between the genetic patterns of different disease types.

\section{Acknowledgements}

Not applicable.

\section{Funding}

The study was supported by the Primary Research \& Development Plan of Jiangsu Province (BE2016630).

\section{Availability of data and materials}

All data generated or analyzed during this study are included in this published article. A genetic database (name: ASD_GD) has been developed and deposited into an open source 'Bioinformatics Database' (http://database.gousinfo.com). The publicly downloadable version (in Excel file) of the database is available at http:/gousinfo.com/database/Data_Genetic/ASD_ GD.xlsx. The database includes 488 genes (with metric scores; ASD_GD $\rightarrow$ Related Genes), 97 pathways (ASD_GD $\rightarrow$ Related Pathways) and 93 diseases (ASD_GD $\rightarrow$ Related Genes) that are associated with ASD. For each ASD-gene association, there is information concerning the supporting references (ASD_GD $\rightarrow$ Ref for Related Genes), including titles and relevant sentences where the association has been identified. For more information regarding the database, please refer to ASD_GD $\rightarrow$ Database Note, where the update status was also included.

\section{Authors' contributions}

$\mathrm{XC}, \mathrm{HC}, \mathrm{CC}$ and FZ contributed to the study design, data collection, data analysis and interpretation. $\mathrm{XC}, \mathrm{HC}$ and $\mathrm{CC}$ contributed to the writing of the manuscript.

\section{Ethics approval and consent to participate}

Not applicable.

\section{Patient consent to publication}

Not applicable.

\section{Competing interests}

The authors declare that they have no competing interests. 


\section{References}

1. Chaste P and Leboyer M: Autism risk factors: Genes, environment, and gene-environment interactions. Dialogues Clin Neurosci 14: 281-292, 2012.

2. Rutter M: Genetic studies of autism: From the 1970s into the millennium. J Abnorm Child Psychol 28: 3-14, 2000.

3. Aronson M, Hagberg B and Gillberg C: Attention deficits and autistic spectrum problems in children exposed to alcohol during gestation: A follow-up study. Dev Med Child Neurol 39: 583-587, 1997.

4. Kolevzon A, Gross R and Reichenberg A: Prenatal and perinatal risk factors for autism: A review and integration of findings. Arch Pediatr Adolesc Med 161: 326-333, 2007.

5. Minshew NJ: Brief report: Brain mechanisms in autism: Functional and structural abnormalities. J Autism Dev Disord 26 205-209, 1996.

6. Tager-Flusberg H: The origins of social impairments in autism spectrum disorder: Studies of infants at risk. Neural Netw 23 1072-1076, 2010

7. Businaro R, Corsi M, Azzara G, Di Raimo T, Laviola G, Romano E, Ricci L, Maccarrone M, Aronica E, Fuso A, et al: Interleukin-18 modulation in autism spectrum disorders J Neuroinflammation 13: 2, 2016.

8. Hu VW: Subphenotype-dependent disease markers for diagnosis and personalized treatment of autism spectrum disorders. Dis Markers 33: 277-288, 2012.

9. Parker-Athill EC and Tan J: Maternal immune activation and autism spectrum disorder: Interleukin-6 signaling as a key mechanistic pathway. Neurosignals 18: 113-128, 2010.

10. Andari E, Duhamel JR, Zalla T, Herbrecht E, Leboyer M and Sirigu A: Promoting social behavior with oxytocin in high-functioning autism spectrum disorders. Proc Natl Acad Sci USA 107: 4389-4394, 2010.

11. Kolevzon A, Bush L, Wang AT, Halpern D, Frank Y, Grodberg D, Rapaport R, Tavassoli T, Chaplin W, Soorya L, et al: A pilot controlled trial of insulin-like growth factor-1 in children with Phelan-McDermid syndrome. Mol Autism 5: 54, 2014.

12. LoParo D and Waldman ID: The oxytocin receptor gene (OXTR) is associated with autism spectrum disorder: A meta-analysis. Mol Psychiatry 20: 640-646, 2015.

13. Zhou Y, Kaiser T, Monteiro P, Zhang X, Van der Goes MS, Wang D, Barak B, Zeng M, Li C, Lu C, et al: Mice with Shank3 mutations associated with ASD and Schizophrenia display both shared and distinct defects. Neuron 89: 147-162, 2016.

14. Cotney J, Muhle RA, Sanders SJ, Liu L, Willsey AJ, Niu W, Liu W, Klei L, Lei J, Yin J, et al: The autism-associated chromatin modifier CHD8 regulates other autism risk genes during human neurodevelopment. Nat Commun 6: 6404, 2015.

15. King LB, Walum H, Inoue K, Eyrich NW and Young LJ: Variation in the oxytocin receptor gene predicts brain region-specific expression and social attachment. Biol Psychiatry 80: 160-169, 2016.

16. Theoharides TC, Stewart JM, Panagiotidou S and Melamed I: Mast cells, brain inflammation and autism. Eur J Pharmacol 778 96-102, 2016.

17. Higashida H, Yokoyama S, Huang JJ, Liu L, Ma WJ, Akther S, Higashida C, Kikuchi M, Minabe Y and Munesue T: Social memory, amnesia, and autism: Brain oxytocin secretion is regulated by $\mathrm{NAD}^{+}$metabolites and single nucleotide polymorphisms of CD38. Neurochem Int 61: 828-838, 2012.
18. Jiang YH and Ehlers MD: Modeling autism by SHANK gene mutations in mice. Neuron 78: 8-27, 2013.

19. Clipperton-Allen AE and Page DT: Decreased aggression and increased repetitive behavior in Pten haploinsufficient mice. Genes Brain Behav 14: 145-157, 2015.

20. Nikitin A, Egorov S, Daraselia N and Mazo I: Pathway studio - the analysis and navigation of molecular networks. Bioinformatics 19: 2155-2157, 2003.

21. Rindflesch TC, Tanabe L, Weinstein JN and Hunter L: EDGAR: Extraction of drugs, genes and relations from the biomedical literature. Pac Symp Biocomput 2000: 517-528, 2000.

22. Sivachenko AY, Yuryev A, Daraselia N and Mazo I: Molecular networks in microarray analysis. J Bioinform Comput Biol 5: 429-456, 2007.

23. Pyatnitskiy M, Mazo I, Shkrob M, Schwartz E and Kotelnikova E: Clustering gene expression regulators: New approach to disease subtyping. PLoS One 9: e84955, 2014.

24. Vanwong N, Ngamsamut N, Medhasi S, Puangpetch A, Chamnanphon M, Tan-Kam T, Hongkaew Y, Limsila P and Sukasem C: Impact of CYP2D6 polymorphism on steady-state plasma levels of risperidone and 9-hydroxyrisperidone in thai children and adolescents with autism spectrum disorder. J Child Adolesc Psychopharmacol 27: 185-191, 2017.

25. Cheadle C, Cao H, Kalinin A and Hodgkinson J: Advanced literature analysis in a Big Data world. Ann NY Acad Sci 1387: 25-33, 2017.

26. Sanders SJ, Murtha MT, Gupta AR, Murdoch JD, Raubeson MJ, Willsey AJ, Ercan-Sencicek AG, DiLullo NM, Parikshak NN, Stein JL, et al: De novo mutations revealed by whole-exome sequencing are strongly associated with autism. Nature 485 : 237-241, 2012.

27. Katayama Y, Nishiyama M, Shoji H, Ohkawa Y, Kawamura A, Sato T, Suyama M, Takumi T, Miyakawa T and Nakayama KI: CHD8 haploinsufficiency results in autistic-like phenotypes in mice. Nature 537: 675-679, 2016.

28. Supek F, Bošnjak M, Skunca $N$ and Šmuc T: REVIGO summarizes and visualizes long lists of gene ontology terms. PLoS One 6: e21800, 2011.

29. Alabdali A, Al-Ayadhi L and El-Ansary A: Association of social and cognitive impairment and biomarkers in autism spectrum disorders. J Neuroinflammation 11: 4, 2014.

30. Goldstein G, Minshew NJ, Allen DN and Seaton BE: High-functioning autism and schizophrenia: A comparison of an early and late onset neurodevelopmental disorder. Arch Clin Neuropsychol 17: 461-475, 2002.

31. Robinson SJ: Childhood epilepsy and autism spectrum disorders: Psychiatric problems, phenotypic expression, and anticonvulsants. Neuropsychol Rev 22: 271-279, 2012.

This work is licensed under a Creative Commons Attribution-NonCommercial-NoDerivatives 4.0 International (CC BY-NC-ND 4.0) License. 